\title{
All Solutions of the Yang-Baxter-Like Matrix Equation for Nilpotent Matrices of Index Two
}

\author{
Duanmei Zhou (iD) ${ }^{1,2}$ and Jiawen Ding ${ }^{1}$ \\ ${ }^{1}$ College of Mathematics and Computer Science, Gannan Normal University, Ganzhou 341000, China \\ ${ }^{2}$ Department of Mathematics, Shanghai University, Shanghai 200444, China
}

Correspondence should be addressed to Duanmei Zhou; gzzdm2008@163.com

Received 14 January 2020; Accepted 2 June 2020; Published 18 June 2020

Academic Editor: Sigurdur F. Hafstein

Copyright (C) 2020 Duanmei Zhou and Jiawen Ding. This is an open access article distributed under the Creative Commons Attribution License, which permits unrestricted use, distribution, and reproduction in any medium, provided the original work is properly cited.

Let $A$ be a nilpotent matrix of index two, and consider the Yang-Baxter-like matrix equation $A X A=X A X$. We first obtain a system of matrix equations of smaller sizes to find all the solutions of the original matrix equation. When $A$ is a nilpotent matrix with rank 1 and rank 2, we get all solutions of the Yang-Baxter-like matrix equation.

\section{Introduction}

We are interested in finding all solutions of the quadratic matrix equation:

$$
A X A=X A X,
$$

where the given $A$ and the unknown $X$ are $n \times n$ square complex matrices with $n \geq 2$. The above equation (1) is called the Yang-Baxter-like matrix equation because it is similar to the classical parameter-free Yang-Baxter equation $[1,2]$. The Yang-Baxter equation was first introduced by Yang in 1967 and then by Baxter in 1972 in the study of statistical mechanics. The Yang-Baxter equation has been extensively researched by mathematicians and physicists in knot theory, braid group theory, and so on [3-6].

Obviously, the Yang-Baxter matrix equation has two trivial solutions $X=0$ and $X=A$. However, we are interested in finding nontrivial solutions. Finding all solutions of equation (1) is a hard work for a general matrix $A$. Indeed, we can reformulate (1) into a system of polynomial equations, so it is equivalent to solving a system of $n^{2}$ quadratic polynomial equations in $n^{2}$ variables. To find all solutions is not an easy task even if for a $3 \times 3$ matrix [7]. Most solutions obtained so far are commuting ones for particular choices of matrices $A$. See, for example, $[8]$ for diagonalizable matrix and $[9,10]$ for nilpotent matrix. In [11], infinitely many solutions of (1) were obtained for any semisimple eigenvalues of the given matrix. A family of commuting solutions of (1) were constructed for those eigenvalues of $A$ that are non-semisimple in $[12,13]$. Some researchers have also proposed some numerical methods for finding commuting solutions. For instance, in [14], when $A$ is a nonsingular matrix such that its inverse is a stochastic matrix, Ding and Rhee found nontrivial solutions of (1) via Brouwer's fixed point theorem. In [15], when $A$ is a diagonalisable matrix, the authors proposed numerical methods to calculate solutions of (1) by applying the mean ergodic theorem. When $A$ is a low rank matrix, all solutions of (1) have been found in [16-18] for the noncommuting case. In [19], the authors have obtained explicit solutions when $A$ is an idempotent matrix. However, for a general matrix $A$, it is difficult to characterize and determine all the solutions of (1), even if for nilpotent matrix.

The purpose of this paper is to find all the solutions of equation (1) under the assumption that $A$ is a nilpotent matrix of index 2 . We first give a system of matrix equations of smaller sizes to find all solutions of the original matrix equation in Section 2. In the next two sections, we study all solutions for $A X A=A X A$ when $A$ is rank one and rank two, respectively. Finally, we present two examples of our solution result in Section 5 and conclude with Section 6. 


\section{All Solutions for the Nilpotent Matrix of Index 2}

Let $A \neq 0$ be a nilpotent matrix with index 2. Clearly, matrix $A$ has 0 as its only eigenvalue and the minimal polynomial of $A$ is $m(t)=t^{2}$. So, we can write the Jordan canonical form $J$ of $A$ as

$$
J=\left[\begin{array}{llll}
0 & & & \\
& J_{0} & & \\
& & \ddots & \\
& & & J_{0}
\end{array}\right],
$$

in which the $2 \times 2$ Jordan block

$$
J_{0}=\left[\begin{array}{ll}
0 & 1 \\
0 & 0
\end{array}\right]
$$

appears $k$ times with $k$ the rank of $A$, and 0 denotes the $(n-2 k) \times(n-2 k)$ zero matrix. So, there exists a nonsingular matrix $W$ such that

$$
W^{-1} A W=J \equiv \operatorname{diag}\left(0, J_{0}, \ldots, J_{0}\right) .
$$

Lemma 1. Let two matrices $A$ and $X$ satisfy $A X A=X A X$. Then, for any nonsingular matrix $U$, the matrices $B=U^{-1} A U$ and $Y=U^{-1} X U$ satisfy

$$
B Y B=Y B Y .
$$

Conversely, if $Y$ satisfies the above equations for a given $B=U^{-1} A U$, then $X=U Y U^{-1}$ satisfies $A X A=X A X$.

According to Lemma 1, we know that solving (1) can be reduced to solving the simplified matrix equation:

$$
Y J Y=J Y J \text {. }
$$

So, solution $X$ to (1) can be expressed as $X=W Y W^{-1}$, where $W$ satisfies $A=W J W^{-1}$. Thus, in our analysis below, we find all solutions of (6). Let $Y$ be partitioned the same way as $J$ into the $(k+1) \times(k+1)$ block matrix:

$$
Y=\left[\begin{array}{cccc}
M & Q_{1} & \cdots & Q_{k} \\
P_{1} & Y_{11} & \cdots & Y_{1 k} \\
\vdots & \vdots & \ddots & \vdots \\
P_{k} & Y_{k 1} & \cdots & Y_{k k}
\end{array}\right],
$$

where $M$ is $(n-2 k) \times(n-2 k), Q_{i}=\left[q_{2 i-1}, q_{2 i}\right] \in \mathbb{C}^{(n-2 k) \times 2}$ and $P_{i}=\left[p_{2 i-1}, p_{2 i}\right]^{T} \in \mathbb{C}^{2 \times(n-2 k)}$ for $i=1, \ldots, k$, and

$$
Y_{i j}=\left[\begin{array}{cc}
b_{i j} & c_{i j} \\
s_{i j} & t_{i j}
\end{array}\right], \quad i, j=1,2, \ldots, k .
$$

Then, the equation $J Y J=Y J Y$ becomes

$$
\left[\begin{array}{cccc}
0 & 0 & \cdots & 0 \\
0 & J_{0} Y_{11} J_{0} & \cdots & J_{0} Y_{1 k} J_{0} \\
\vdots & \vdots & \ddots & \vdots \\
0 & J_{0} Y_{k 1} J_{0} & \vdots & J_{0} Y_{k k} J_{0}
\end{array}\right]=\left[\begin{array}{ccccc}
\sum_{i=1}^{k} Q_{i} J_{0} P_{i} & \sum_{i=1}^{k} Q_{i} J_{0} Y_{i 1} & \cdots & \sum_{i=1}^{k} Q_{i} J_{0} Y_{i k} \\
\sum_{i=1}^{k} Y_{1 i} J_{0} P_{i} & \sum_{i=1}^{k} Y_{1 i} J_{0} Y_{i 1} & \cdots & \sum_{i=1}^{k} Y_{1 i} J_{0} Y_{i k} \\
\vdots & \vdots & \ddots & \vdots \\
\sum_{i=1}^{k} Y_{k i} J_{0} P_{i} & \sum_{i=1}^{k} Y_{k i} J_{0} Y_{i 1} & \cdots & \sum_{i=1}^{k} Y_{k i} J_{0} Y_{i k}
\end{array}\right]
$$

which is equivalent to the system

$$
\begin{cases}\sum_{i=1}^{k} Q_{i} J_{0} P_{i}=0, & \\ \sum_{i=1}^{k} Y_{j i} J_{0} P=0, & j=1,2, \ldots, k,_{i} \\ \sum_{i=1}^{k} Q_{i} J_{0} Y_{i j}=0, & j=1,2, \ldots, k \\ \sum_{i=1}^{k} Y_{l i} J_{0} Y_{i j}=J_{0} Y_{l j} J_{0}, & l, j=1,2, \ldots, k\end{cases}
$$

Note that the above system does not contain $M$. This means that $M$ is arbitrary. Because finding all solutions of (10) is very difficult, we focus on finding all solutions of (10) when $k=1$ and $k=2$.

\section{All Solutions for the Nilpotent Matrix of Index 2 with Rank 1}

When $k=1$, this means that the $\operatorname{rank}$ of $A$ is 1 . Then,

$$
J=\left[\begin{array}{ll}
0 & 0 \\
0 & J_{0}
\end{array}\right],
$$

where 0 is the $(n-2) \times(n-2)$ zero matrix. Let $Y$ be partitioned the same way as $J$ into the $2 \times 2$ block matrix:

$$
Y=\left[\begin{array}{cc}
M & Q \\
P & Z
\end{array}\right],
$$

where $\quad M \in \mathbb{C}^{(n-2) \times(n-2)}, \quad Q=\left[\begin{array}{ll}q_{1} & q_{2}\end{array}\right] \in \mathbb{C}^{(n-2) \times 2}, \quad P=$ $\left[\begin{array}{ll}p_{1} & p_{2}\end{array}\right]^{T} \in \mathbb{C}^{2 \times(n-2)}$, and $Z \in \mathbb{C}^{2 \times 2}$. According to (4), we obtain

$$
\left\{\begin{array}{l}
Q J_{0} P=0, \\
Q J_{0} Z=0, \\
Z J_{0} P=0, \\
Z J_{0} Z=J_{0} Z J_{0} .
\end{array}\right.
$$

Obviously, the last equation of (13) is also a Yang-Baxter matrix equation. Next, we solve the last equation in (13).

Lemma 2. The solutions $Z$ of the equation $Z J_{0} Z=J_{0} Z J_{0}$ are 


$$
\begin{aligned}
& Z^{(1)}=\left[\begin{array}{ll}
0 & z_{2} \\
0 & z_{4}
\end{array}\right], \quad z_{4} \neq 0, \\
& Z^{(2)}=\left[\begin{array}{ll}
0 & z_{2} \\
0 & 0
\end{array}\right], \\
& Z^{(3)}=\left[\begin{array}{cc}
z_{1} & z_{2} \\
0 & 0
\end{array}\right], \quad z_{1} \neq 0 .
\end{aligned}
$$

Proof. Let

$$
Z=\left[\begin{array}{ll}
z_{1} & z_{2} \\
z_{3} & z_{4}
\end{array}\right]
$$

Then, the equation $Z J_{0} Z=J_{0} Z J_{0}$ becomes

$$
\left[\begin{array}{ll}
z_{1} & z_{2} \\
z_{3} & z_{4}
\end{array}\right]\left[\begin{array}{ll}
0 & 1 \\
0 & 0
\end{array}\right]\left[\begin{array}{ll}
z_{1} & z_{2} \\
z_{3} & z_{4}
\end{array}\right]=\left[\begin{array}{ll}
0 & 1 \\
0 & 0
\end{array}\right]\left[\begin{array}{ll}
z_{1} & z_{2} \\
z_{3} & z_{4}
\end{array}\right]\left[\begin{array}{ll}
0 & 1 \\
0 & 0
\end{array}\right] .
$$

That is,

$$
\left[\begin{array}{cc}
z_{1} z_{3} & z_{1} z_{4} \\
z_{3}^{2} & z_{3} z_{4}
\end{array}\right]=\left[\begin{array}{cc}
0 & z_{3} \\
0 & 0
\end{array}\right]
$$

This leads to $z_{3}=0$ and $z_{4} z_{1}=0$, and $z_{2}$ is arbitrary. By Lemma 2, all solutions of the last equation of (13) are $Z^{(1)}, Z^{(2)}$, and $Z^{(3)}$. Substituting such matrices into the first three equations of (13), we obtain the following result.

Theorem 1. Suppose that $A$ is a nilpotent matrix with rank 1. Then, all solutions of (1) are $X=W Y W^{-1}$, where $Y$ is partitioned as (12) in which $M$ is an arbitrary $(n-2) \times(n-2)$ matrix such that $Y=$

$$
\begin{aligned}
& {\left[\begin{array}{ccc}
M & 0 & q_{2} \\
p_{1}^{T} & 0 & z_{2} \\
p_{2}^{T} & 0 & z_{4}
\end{array}\right], \quad z_{4} \neq 0,} \\
& {\left[\begin{array}{ccc}
M & 0 & q_{2} \\
p_{1}^{T} & 0 & z_{2} \\
p_{2}^{T} & 0 & 0
\end{array}\right],} \\
& {\left[\begin{array}{ccc}
M & q_{1} & q_{2} \\
p_{1}^{T} & 0 & z_{2} \\
0 & 0 & 0
\end{array}\right],} \\
& {\left[\begin{array}{ccc}
M & q_{1} & q_{2} \\
p_{1}^{T} & z_{1} & z_{2} \\
0 & 0 & 0
\end{array}\right], \quad z_{1} \neq 0 .}
\end{aligned}
$$

Proof. When $Z=Z^{(1)}$, then $Z J_{0}=0$. So, we just need to solve the first two equations in (13). The second equation (13) is $Q J_{0} Z=0$. That is,

$$
\left[\begin{array}{ll}
0 & q_{1} z_{4}
\end{array}\right]=0 \text {. }
$$
(18).

Since $z_{4} \neq 0$, we have $q_{1}=0$. Thus, $Q J_{0}=0$. This gives

Let $Z=Z^{(2)}$. Then, $Z J_{0}=0$ and $J_{0} Z=0$. So, the second and the third equations of (13) are satisfied. Next, we only have to solve the first equation of (13), i.e.,

$$
Q J_{0} P=\left[\begin{array}{ll}
q_{1} & q_{2}
\end{array}\right]\left[\begin{array}{ll}
0 & 1 \\
0 & 0
\end{array}\right]\left[\begin{array}{c}
p_{1}^{T} \\
p_{2}^{T}
\end{array}\right]=q_{1} p_{2}^{T}=0,
$$

from which $q_{1}=0, p_{2}$ is arbitrary or $p_{2}=0, q_{1}$ is arbitrary. This gives (19).

If $Z=Z^{(3)}$, then $J_{0} Z=0$. So, the second equation of (13) is satisfied. From the first equation $Z J_{0} P=0$, we obtain

$$
\left[\begin{array}{c}
z_{1} p_{2}^{T} \\
0
\end{array}\right]=0
$$

which leads to $z_{1} p_{2}^{T}=0$. Since $z_{1} \neq 0$, we obtain $p_{2}=0$. Thus, $J_{0} P=0$. This gives (20).

\section{All Solutions for the Nilpotent Matrix of Index 2 with Rank 2}

When $k=2$, this means

$$
J=\left[\begin{array}{ccc}
0 & 0 & 0 \\
0 & J_{0} & 0 \\
0 & 0 & J_{0}
\end{array}\right],
$$

where 0 is the $(n-4) \times(n-4)$ zero matrix. Let $\Lambda=\left[\begin{array}{cc}J_{0} & 0 \\ 0 & J_{0}\end{array}\right]$. Then,

$$
J=\left[\begin{array}{ll}
0 & 0 \\
0 & \Lambda
\end{array}\right]
$$

Let $Y$ be partitioned the same way as $J$ into the $2 \times 2$ block matrix:

$$
Y=\left[\begin{array}{cc}
M & U \\
V^{T} & K
\end{array}\right]
$$

where $M$ is $(n-4) \times(n-4), U=\left[u_{1}, u_{2}, u_{3}, u_{4}\right] \epsilon$ $\mathbb{C}^{(n-4) \times 4}, V=\left[v_{1}, v_{2}, v_{3}, v_{4},\right] \in \mathbb{C}^{(n-4) \times 4}$, and $K$ is $4 \times 4$. Then, $J Y J=Y J Y$ is equivalent to the system:

$$
\left\{\begin{array}{l}
U \Lambda V^{T}=0, \\
U \Lambda K=0, \\
K \Lambda V^{T}=0, \\
\Lambda K \Lambda=K \Lambda K .
\end{array}\right.
$$

All solutions of this system were obtained in [18]. We have the following results.

Lemma 3 (see Lemma 4.1 in [18]). The solutions $K$ of the equation $\Lambda K \Lambda=K \Lambda K$ are 


$$
\begin{aligned}
& K_{1}=\left[\begin{array}{llll}
0 & a_{12} & 0 & a_{14} \\
0 & a_{22} & 0 & a_{24} \\
0 & a_{32} & 0 & a_{34} \\
0 & a_{42} & 0 & a_{44}
\end{array}\right], \\
& K_{2}=\left[\begin{array}{cccc}
0 & a_{12} & 0 & a_{14} \\
0 & a_{22} & 0 & a_{24} \\
0 & a_{32} & a_{33} & a_{34} \\
0 & 0 & 0 & 0
\end{array}\right], \quad a_{33} \neq 0, \\
& K_{3}=\left[\begin{array}{cccc}
0 & a_{12} & a_{13} & a_{14} \\
0 & a_{22} & 0 & a_{24} \\
0 & a_{32} & a_{33} & a_{34} \\
0 & 0 & 0 & 0
\end{array}\right], \quad a_{13} \neq 0 \\
& K_{4}=\left[\begin{array}{cccc}
0 & a_{12} & a_{13} & a_{14} \\
0 & 0 & 0 & 0 \\
a_{31} & a_{32} & a_{33} & a_{34} \\
0 & 0 & 0 & 0
\end{array}\right], \quad a_{13} a_{31} \neq 0, \\
& K_{5}=\left[\begin{array}{cccc}
0 & a_{12} & 0 & a_{14} \\
0 & -\frac{a_{33} a_{42}}{a_{31}} & 0 & -\frac{a_{33} a_{44}}{a_{31}} \\
a_{31} & a_{32} & a_{33} & a_{34} \\
0 & a_{42} & 0 & a_{44}
\end{array}\right], \quad a_{31} \neq 0, \\
& K_{6}=\left[\begin{array}{cccc}
a_{11} & a_{12} & a_{13} & a_{14} \\
0 & -\frac{a_{13} a_{42}}{a_{11}} & 0 & -\frac{a_{13} a_{44}}{a_{11}} \\
a_{31} & a_{32} & \frac{a_{13} a_{31}}{a_{11}} & a_{34} \\
0 & a_{42} & 0 & a_{44}
\end{array}\right], \quad a_{11} \neq 0, \\
& K_{7}=\left[\begin{array}{cccc}
a_{11} & a_{12} & a_{13} & a_{14} \\
0 & 0 & 0 & 0 \\
a_{31} & a_{32} & a_{33} & a_{34} \\
0 & 0 & 0 & 0
\end{array}\right], \quad a_{11} \neq 0,\left|\begin{array}{ll}
a_{11} & a_{13} \\
a_{31} & a_{33}
\end{array}\right| \neq 0 .
\end{aligned}
$$

Theorem 2 (see Theorem 4.2 in [18]). Suppose $A$ is a nilpotent matrix with rank 2 , and is partitioned as (4). Then, all solutions of (1) are $X=W Y W^{-1}$, where $Y$ is partitioned as
(10) in which $M$ is an arbitrary $(n-4) \times(n-4)$ matrix such that $Y=$

$$
\left[\begin{array}{ccccc}
M & 0 & u_{2} & 0 & u_{4} \\
v_{1}^{T} & 0 & a_{12} & 0 & a_{14} \\
v_{2} T & 0 & a_{22} & 0 & a_{24} \\
v_{3}^{T} & 0 & a_{32} & 0 & a_{34} \\
v_{4}^{T} & 0 & a_{42} & 0 & a_{44}
\end{array}\right],\left[\begin{array}{ccccc}
M & 0 & u_{2} & u_{3} & u_{4} \\
v_{1}^{T} & 0 & a_{12} & 0 & a_{14} \\
v_{2} T & 0 & a_{22} & 0 & a_{24} \\
v_{3}^{T} & 0 & a_{32} & 0 & a_{34} \\
0^{T} & 0 & 0 & 0 & 0
\end{array}\right], \quad u_{3} \neq 0
$$$$
\left[\begin{array}{ccccc}
M & u_{1} & u_{2} & u_{3} & u_{4} \\
v_{1}^{T} & 0 & a_{12} & 0 & a_{14} \\
-\frac{u_{1}^{H} u_{3}}{\left\|u_{1}\right\|^{2}} v_{4}^{T} & 0 & -\frac{u_{1}^{H} u_{3}}{\left\|u_{1}\right\|^{2}} a_{42} & 0 & -\frac{u_{1}^{H} u_{3}}{\left\|u_{1}\right\|^{2}} a_{44} \\
v_{3}^{T} & 0 & a_{32} & 0 & a_{34} \\
v_{4}^{T} & 0 & a_{42} & 0 & a_{44}
\end{array}\right], \quad u_{1} \neq 0
$$$$
\left[\begin{array}{ccccc}
M & 0 & u_{2} & u_{3} & u_{4} \\
v_{1}^{T} & 0 & a_{12} & 0 & a_{14} \\
v_{2}^{T} & 0 & a_{22} & 0 & a_{24} \\
v_{3}^{T} & 0 & a_{32} & a_{33} & a_{34} \\
0^{T} & 0 & 0 & 0 & 0
\end{array}\right],\left[\begin{array}{ccccc}
M & u_{1} & u_{2} & u_{3} & u_{4} \\
v_{1}^{T} & 0 & a_{12} & 0 & a_{14} \\
0^{T} & 0 & 0 & 0 & 0 \\
v_{3}^{T} & 0 & a_{32} & a_{33} & a_{34} \\
0^{T} & 0 & 0 & 0 & 0
\end{array}\right], \quad u_{1} \neq 0, a_{33} \neq 0,
$$

$$
\left[\begin{array}{ccccc}
M & 0 & u_{2} & u_{3} & u_{4} \\
v_{1}^{T} & 0 & a_{12} & a_{13} & a_{14} \\
v_{2}^{T} & 0 & a_{22} & 0 & a_{24} \\
v_{3}^{T} & 0 & a_{32} & a_{33} & a_{34} \\
0^{T} & 0 & 0 & 0 & 0
\end{array}\right]
$$$$
\left[\begin{array}{ccccc}
M & u_{1} & u_{2} & u_{3} & u_{4} \\
v_{1}^{T} & 0 & a_{12} & a_{13} & a_{14} \\
0^{T} & 0 & 0 & 0 & 0 \\
v_{3}^{T} & 0 & a_{32} & a_{33} & a_{34} \\
0^{T} & 0 & 0 & 0 & 0
\end{array}\right], \quad u_{1} \neq 0, a_{13} \neq 0
$$$$
\left[\begin{array}{ccccc}
M & u_{1} & u_{2} & u_{3} & u_{4} \\
v_{1}^{T} & 0 & a_{12} & a_{13} & a_{14} \\
0^{T} & 0 & 0 & 0 & 0 \\
v_{3}^{T} & a_{31} & a_{32} & a_{33} & a_{34} \\
0^{T} & 0 & 0 & 0 & 0
\end{array}\right],\left[\begin{array}{ccccc}
M & u_{1} & u_{2} & \frac{a_{33}}{a_{31}} u_{1} & u_{4} \\
v_{1}^{T} & 0 & a_{12} & 0 & a_{14} \\
-\frac{a_{33}}{a_{31}} v_{4}^{T} & 0 & -\frac{a_{33} a_{42}}{a_{31}} & 0 & -\frac{a_{33} a_{44}}{a_{31}} \\
v_{3}^{T} & a_{31} & a_{32} & a_{33} & a_{34} \\
v_{4}^{T} & 0 & a_{42} & 0 & a_{44}
\end{array}\right], \quad a_{13} \neq, a_{31} \neq 0,
$$$$
\left[\begin{array}{ccccc}
M & u_{1} & u_{2} & u_{3} & u_{4} \\
v_{1}^{T} & 0 & a_{12} & 0 & a_{14} \\
0^{T} & 0 & 0 & 0 & 0 \\
v_{3}^{T} & a_{31} & a_{32} & a_{33} & a_{34} \\
0^{T} & 0 & 0 & 0 & 0
\end{array}\right],\left[\begin{array}{ccccc}
M & u_{1} & u_{2} & u_{3} & u_{4} \\
v_{1}^{T} & a_{11} & a_{12} & a_{13} & a_{14} \\
0^{T} & 0 & 0 & 0 & 0 \\
v_{3}^{T} & a_{31} & a_{32} & \frac{a_{13} a_{31}}{a_{11}} & a_{34} \\
0^{T} & 0 & 0 & 0 & 0
\end{array}\right],
$$ 
with $a_{13} \neq 0, u_{3} \neq a_{33} u_{1} / a_{31}$ in the left matrix, and $a_{11} \neq 0$, $u_{3} \neq a_{13} u_{1} / a_{11}$ in the second one,

$$
\begin{aligned}
& {\left[\begin{array}{ccccc}
M & u_{1} & u_{2} & \frac{a_{13}}{a_{11}} u_{1} & u_{4} \\
v_{1}^{T} & a_{11} & a_{12} & a_{13} & a_{14} \\
-\frac{a_{13}}{a_{11}} v_{4}^{T} & 0 & -\frac{a_{13} a_{42}}{a_{11}} & 0 & -\frac{a_{13} a_{44}}{a_{11}} \\
v_{3}^{T} & a_{31} & a_{32} & \frac{a_{13} a_{31}}{a_{11}} & a_{34} \\
v_{4}^{T} & 0 & a_{42} & 0 & a_{44}
\end{array}\right],} \\
& {\left[\begin{array}{ccccc}
M & u_{1} & u_{2} & u_{3} & u_{4} \\
v_{1}^{T} & a_{11} & a_{12} & a_{13} & a_{14} \\
0^{T} & 0 & 0 & 0 & 0 \\
v_{3}^{T} & a_{31} & a_{32} & a_{33} & a_{34} \\
0^{T} & 0 & 0 & 0 & 0
\end{array}\right],} \\
& a_{11} \neq 0, \\
& a_{33} \neq \frac{a_{13} a_{31}}{a_{11}} .
\end{aligned}
$$

\section{Examples}

We give two examples to illustrate our results.

Example 1. Let

$$
A=\left[\begin{array}{ccc}
1 & 0 & 1 \\
1 & 0 & 1 \\
-1 & 0 & -1
\end{array}\right] .
$$

Then, there exists

$$
\begin{aligned}
W & =\left[\begin{array}{ccc}
1 & 1 & 0 \\
0 & 1 & -1 \\
-1 & -1 & 1
\end{array}\right], \\
J & =\left[\begin{array}{lll}
0 & 0 & 0 \\
0 & 0 & 1 \\
0 & 0 & 0
\end{array}\right],
\end{aligned}
$$

such that $A=W J W^{-1}$. By Theorem 1, we obtain all solutions of (1) as

$$
\begin{aligned}
& X=\left[\begin{array}{ccc}
q_{2}+z_{2} & -m-p_{1} & -m-p_{1}+q_{2}+z_{2} \\
z_{2}-z_{4} & -p_{1}+p_{2} & -p_{1}+p_{2}+z_{2}-z_{4} \\
-q_{2}-z_{2}+z_{4} & m+p_{1}-p_{2} & m+p_{1}-p_{2}-q_{2}-z_{2}+z_{4}
\end{array}\right], \quad z_{4} \neq 0, \\
& X=\left[\begin{array}{ccc}
q_{2}+z_{2} & -m-p_{1} & -m-p_{1}+q_{2}+z_{2} \\
z_{2} & -p_{1}+p_{2} & -p_{1}+p_{2}+z_{2} \\
-q_{2}-z_{2} & m+p_{1}-p_{2} & m+p_{1}-p_{2}-q_{2}-z_{2}
\end{array}\right], \\
& X=\left[\begin{array}{ccc}
q_{1}+q_{2}+z_{2} & q_{1}-p_{1}-m & q_{1}-p_{1}-m+q_{2}+z_{2} \\
z_{2} & -p_{1} & z_{2}-p_{1} \\
-q_{1}-q_{2}-z_{2} & m+p_{1}-q_{1} & m+p_{1}-q_{1}-q_{2}-z_{2}
\end{array}\right], \\
& X=\left[\begin{array}{ccc}
q_{1}+q_{2}+z_{1}+z_{2} & q_{1}-p_{1}-m+z_{1} & q_{1}-p_{1}-m+q_{2}+z_{1}+z_{2} \\
z_{1}+z_{2} & z_{1}-p_{1} & z_{1}-p_{1}+z_{2} \\
-q_{1}-q_{2}-z_{1}-z_{2} & m+p_{1}-q_{1}-z_{1} & m+p_{1}-q_{1}-q_{2}-z_{1}-z_{2}
\end{array}\right], z_{1} \neq 0 .
\end{aligned}
$$

The second example is a matrix with rank 2 .

Example 2. Let

$$
A=\left[\begin{array}{lllll}
0 & 0 & 0 & 0 & 0 \\
0 & 0 & 0 & 1 & 0 \\
0 & 0 & 0 & 0 & 1 \\
0 & 0 & 0 & 0 & 0 \\
0 & 0 & 0 & 0 & 0
\end{array}\right]=W J W^{-1}
$$

$$
=\left[\begin{array}{lllll}
1 & 0 & 0 & 0 & 0 \\
0 & 1 & 0 & 0 & 0 \\
0 & 0 & 0 & 1 & 0 \\
0 & 0 & 1 & 0 & 0 \\
0 & 0 & 0 & 0 & 1
\end{array}\right]\left[\begin{array}{lllll}
0 & 0 & 0 & 0 & 0 \\
0 & 0 & 1 & 0 & 0 \\
0 & 0 & 0 & 0 & 0 \\
0 & 0 & 0 & 0 & 1 \\
0 & 0 & 0 & 0 & 0
\end{array}\right]\left[\begin{array}{lllll}
1 & 0 & 0 & 0 & 0 \\
0 & 1 & 0 & 0 & 0 \\
0 & 0 & 0 & 1 & 0 \\
0 & 0 & 1 & 0 & 0 \\
0 & 0 & 0 & 0 & 1
\end{array}\right] .
$$

All solutions $Y$ of $(11)$ are $K_{1}, \ldots, K_{7}$ as given by Lemma 3 . Multiplying $X=W Y W^{-1}=W K_{k} W^{-1}$ out with $k=1, \ldots, 7$, we find all solutions of (1) are 
$X=\left[\begin{array}{lllll}m & 0 & 0 & u_{2} & u_{4} \\ v_{1} & 0 & 0 & a_{12} & a_{14} \\ v_{3} & 0 & 0 & a_{32} & a_{34} \\ v_{2} & 0 & 0 & a_{22} & a_{24} \\ v_{4} & 0 & 0 & a_{42} & a_{44}\end{array}\right],\left[\begin{array}{ccccc}m & 0 & u_{3} & u_{2} & u_{4} \\ v_{1} & 0 & 0 & a_{12} & a_{14} \\ v_{3} & 0 & 0 & a_{32} & a_{34} \\ v_{2} & 0 & 0 & a_{22} & a_{24} \\ 0 & 0 & 0 & 0 & 0\end{array}\right], \quad u_{3} \neq 0$,

$X=\left[\begin{array}{ccccc}m & u_{1} & u_{2} & u_{3} & u_{4} \\ v_{1} & 0 & 0 & a_{12} & a_{14} \\ v_{3} & 0 & 0 & a_{32} & a_{34} \\ -\frac{u_{1}^{H} u_{3}}{\left\|u_{1}\right\|^{2}} v_{4}^{T} & 0 & 0 & -\frac{u_{1}^{H} u_{3}}{\left\|u_{1}\right\|^{2}} a_{42} & -\frac{u_{1}^{H} u_{3}}{\left\|u_{1}\right\|^{2}} a_{44} \\ v_{4}^{T} & 0 & 0 & a_{42} & a_{44}\end{array}\right], \quad u_{1} \neq 0$,

$X=\left[\begin{array}{ccccc}m & 0 & u_{3} & u_{2} & u_{4} \\ v_{1} & 0 & 0 & a_{12} & a_{14} \\ v_{3} & 0 & a_{33} & a_{32} & a_{34} \\ v_{2} & 0 & 0 & a_{22} & a_{24} \\ 0 & 0 & 0 & 0 & 0\end{array}\right],\left[\begin{array}{ccccc}m & u_{1} & u_{2} & u_{3} & u_{4} \\ v_{1} & 0 & 0 & a_{12} & a_{14} \\ v_{3} & 0 & a_{33} & a_{32} & a_{34} \\ 0 & 0 & 0 & 0 & 0 \\ 0 & 0 & 0 & 0 & 0\end{array}\right], \quad u_{1} \neq 0, a_{33} \neq 0$,

$X=\left[\begin{array}{ccccc}m & 0 & u_{3} & u_{2} & u_{4} \\ v_{1} & 0 & a_{13} & a_{12} & a_{14} \\ v_{3} & 0 & a_{33} & a_{32} & a_{34} \\ v_{2} & 0 & 0 & a_{22} & a_{24} \\ 0 & 0 & 0 & 0 & 0\end{array}\right],\left[\begin{array}{ccccc}m & u_{1} & u_{3} & u_{2} & u_{4} \\ v_{1} & 0 & a_{13} & a_{12} & a_{14} \\ v_{3} & 0 & a_{33} & a_{32} & a_{34} \\ 0 & 0 & 0 & 0 & 0 \\ 0 & 0 & 0 & 0 & 0\end{array}\right], \quad u_{1} \neq 0, a_{13} \neq 0$,

$X=\left[\begin{array}{ccccc}m & u_{1} & u_{3} & u_{2} & u_{4} \\ v_{1} & 0 & a_{13} & a_{12} & a_{14} \\ v_{3} & a_{31} & a_{33} & a_{32} & a_{34} \\ 0 & 0 & 0 & 0 & 0 \\ 0 & 0 & 0 & 0 & 0\end{array}\right],\left[\begin{array}{ccccc}m & u_{1} & \frac{a_{33}}{a_{31}} u_{1} & u_{2} & u_{4} \\ v_{1} & 0 & 0 & a_{12} & a_{14} \\ v_{3} & a_{31} & a_{33} & a_{32} & a_{34} \\ -a_{33} & 0 & 0 & -\frac{a_{33} a_{42}}{a_{31}} & -\frac{a_{33} a_{44}}{a_{31}} \\ v_{3} & a_{31} & a_{32} & a_{33} & a_{34} \\ v_{4} & 0 & 0 & a_{42} & a_{44}\end{array}\right]$,

$X=\left[\begin{array}{ccccc}m & u_{1} & u_{3} & u_{2} & u_{4} \\ v_{1} & 0 & 0 & a_{12} & a_{14} \\ v_{3} & a_{31} & a_{33} & a_{32} & a_{34} \\ 0 & 0 & 0 & 0 & 0 \\ 0 & 0 & 0 & 0 & 0\end{array}\right],\left[\begin{array}{ccccc}m & u_{1} & u_{3} & u_{2} & u_{4} \\ v_{1} & a_{11} & a_{13} & a_{12} & a_{14} \\ v_{3}^{T} & a_{31} & \frac{a_{13} a_{31}}{a_{11}} & a_{32} & a_{34} \\ 0 & 0 & 0 & 0 & 0 \\ 0 & 0 & 0 & 0 & 0\end{array}\right]$, with $a_{13} \neq 0, \quad u_{3} \neq a_{33} u_{1} / a_{3} 1$ in the left matrix, and $a_{11} \neq 0 u_{3} \neq a_{13} u_{1} / a_{11}$ in the second one,

$$
\begin{aligned}
& X=\left[\begin{array}{ccccc}
m & u_{1} & \frac{a_{13}}{a_{11}} u_{1} & u_{2} & u_{4} \\
v_{1} & a_{11} & a_{13} & a_{12} & a_{14} \\
v_{3} & a_{31} & \frac{a_{13} a_{31}}{a_{11}} & a_{32} & a_{34} \\
-\frac{a_{13}}{a_{11}} v_{4}^{T} & 0 & 0 & -\frac{a_{13} a_{42}}{a_{11}} & -\frac{a_{13} a_{44}}{a_{1} 1} \\
v_{4} & 0 & 0 & a_{42} & a_{44}
\end{array}\right], \\
& {\left[\begin{array}{ccccc}
m & u_{1} & u_{3} & u_{2} & u_{4} \\
v_{1} & a_{11} & a_{13} & a_{12} & a_{14} \\
v_{3} & a_{31} & a_{33} & a_{32} & a_{34} \\
0 & 0 & 0 & 0 & 0 \\
0 & 0 & 0 & 0 & 0
\end{array}\right],} \\
& a_{11} \neq 0, \\
& a_{33} \neq \frac{a_{13} a_{31}}{a_{11}} .
\end{aligned}
$$

\section{Conclusions}

In this paper, we obtain all solutions for the Yang-Baxterlike (1) when $A$ is a nilpotent matrix of index 2. We first obtain a system of matrix equations of smaller sizes to find all the solutions of the original matrix equation. For a special case, that is, $k=1$ and $k=2$, we derive all solutions of the Yang-Baxter-like matrix equation in detail. However, for an arbitrary nilpotent matrix, finding all solutions of (1) is a hard work. We hope to solve this problem in the future.

\section{Conflicts of Interest}

The authors declare that there are no conflicts of interest regarding the publication of this paper.

\section{Acknowledgments}

This work was supported by the National Natural Science Foundation of China (no. 11861008), China Postdoctoral Science Foundation (no. 2018M641974), Natural Science Foundation of Jiangxi Province (no. 20192BAB201008), China Scholarship Council (no. 201909865004), Research fund of Gannan Normal University (Nos. YJG-2018-11 and 18zb04), and Key Disciplines Coordinate Innovation Projects of Gannan Normal University. 


\section{References}

[1] R. J. Baxter, "Partition function of the Eight-Vertex lattice model," Annals of Physics, vol. 70, no. 1, pp. 193-228, 1972.

[2] C. N. Yang, "Some exact results for the many-body problem in one dimension with repulsive delta-function interaction," Physical Review Letters, vol. 19, no. 23, pp. 1312-1315, 1967.

[3] L. D. Faddeev, "History and perspectives of quantum groups," Milan Journal of Mathematics, vol. 74, no. 1, pp. 279-294, 2006.

[4] F. Felix, Nonlinear Equations, Quantum Groups and Duality Theorems: A Primer on the Yang-Baxter Equation, VDM Verlag, Saarbrücken, Germany, 2009.

[5] M. Jimbo, Introduction to the Yang-Baxter Equation, Braid Group, Knot Theory and Statistical Physics II, pp. 153-176, World Scientic, Singapore, 1994.

[6] C. Yang and M. Ge, Braid Group, Knot Theory, and Statistical Mechanics, World Scientific, Singapore, 1989.

[7] A. Cibotarica, J. Ding, J. Kolibal, and N. Rhee, "Solutions of the Yang-Baxter matrix equation for an idempotent," $\mathrm{Nu}$ merical Algebra, Control and Optimization Journal, vol. 3, no. 2, pp. 235-245, 2013.

[8] Q. Dong and J. Ding, "Complete commuting solutions of the Yang-Baxter-like matrix equation for diagonalizable matrices," Computers \& Mathematics with Applications, vol. 72, no. 1, pp. 194-201, 2016.

[9] Q. Dong, J. Ding, and Q. Huang, "Commuting solutions of a quadratic matrix equation for nilpotent matrices," Algebra Colloquium, vol. 25, no. 1, pp. 31-44, 2018.

[10] D. Zhou and J. Ding, "Solving the Yang-Baxter-like matrix equation for nilpotent matrices of index three," International Journal of Computer Mathematics, vol. 95, no. 2, pp. 303-315, 2018.

[11] J. Ding and C. Zhang, "On the structure of the spectral solutions of the Yang-Baxter matrix equation," Applied Mathematics Letters, vol. 35, pp. 86-89, 2014.

[12] Q. Dong, "Projection-based commuting solutions of the Yang-Baxter matrix equation for non-semisimple eigenvalues," Applied Mathematics Letters, vol. 64, pp. 231-234, 2017.

[13] D. Zhou, G. Chen, G. Yu, and J. Zhong, "On the projectionbased commuting solutions of the Yang-Baxter matrix equation," Applied Mathematics Letters, vol. 79, pp. 155-161, 2018.

[14] J. Ding and N. H. Rhee, "A nontrivial solution to a stochastic matrix equation," East Asian Journal on Applied Mathematics, vol. 2, no. 4, pp. 277-284, 2012.

[15] J. Ding and N. H. Rhee, "Computing solutions of the YangBaxter-like matrix equation for diagonalisable matrices," East Asian Journal on Applied Mathematics, vol. 5, no. 1, pp. 75-84, 2015.

[16] H. Tian, "All solutions of the Yang-Baxter-like matrix equation for rank-one matrices," Applied Mathematics Letters, vol. 51, pp. 55-59, 2016.

[17] D. Zhou, G. Chen, and J. Ding, "Solving the Yang-Baxter-like matrix equation for rank-two matrices," Journal of Computational and Applied Mathematics, vol. 313, pp. 142-151, 2017.

[18] D. Zhou, G. Chen, and J. Ding, "On the Yang-Baxter-like matrix equation for rank-two matrices," Open Mathematics, vol. 15, no. 1, pp. 340-353, 2017.

[19] S. I. A. Mansour, J. Ding, and Q. Huang, "Explicit solutions of the Yang-Baxter-like matrix equation for an idempotent matrix," Applied Mathematics Letters, vol. 63, pp. 71-76, 2017. 